\title{
Clinical interest of the combined use of serum CD26 and alpha-L-fucosidase in the early diagnosis of colorectal cancer
}

\author{
Daniel Ayude ${ }^{\mathrm{a}}$, María Páez de la Cadena ${ }^{\mathrm{a}}$, Oscar Javier Cordero ${ }^{\mathrm{b}}$, Montserrat Nogueira $^{\mathrm{b}}$, José Ayude $^{\mathrm{c}}$, \\ Almudena Fernández-Briera ${ }^{\mathrm{a}}$ and Francisco Javier Rodríguez-Berrocal ${ }^{\mathrm{a}, *}$ \\ ${ }^{a}$ Department of Biochemistry, Genetics, and Immunology, University of Vigo 36200 Vigo, Spain \\ ${ }^{\mathrm{b}}$ Department of Biochemistry and Molecular Biology, University of Santiago de Compostela, 15706 Santiago de \\ Compostela, Spain \\ ${ }^{\mathrm{c}}$ Department of Computer Sciences, University of Vigo, 36200 Vigo, Spain
}

\begin{abstract}
The purpose of this study was to assess if the combination of CD26 and alpha-L-fucosidase has a role in the diagnosis of colorectal cancer, paying particular attention to the stages in which the tumour is not yet disseminated. CD26 concentration and alpha-L-fucosidase activity were determined in sera from 110 colorectal cancer patients and 46 donors. The combination of CD26 and alpha-L-fucosidase showed a specificity of $100 \%$ with a sensitivity of $64 \%$ in the diagnosis of colorectal cancer. Interestingly, the combination of both markers had a sensitivity of $75 \%$ in the stage I at the highest specificity (100\%), providing also high sensitivity levels for the other non-disseminated stages ( $66 \%$ for stages II and III). In conclusion, the combined use of CD26 and alpha-L-fucosidase offers high sensitivity with high specificity in the diagnosis of colorectal cancer, especially at the earliest stage (TNM I).
\end{abstract}

Keywords: Colorectal cancer, serum, CD26, alpha-L-fucosidase, diagnosis

\section{Introduction}

Colorectal cancer (CRC) still represents the second most frequent cause of cancer death in Western countries [1], despite that it is a carcinoma with higher expectations of survival when diagnosed at early stages. Thus, it seems reasonable to explore the application of new molecular markers for earlier detection of cancer [2].

Due to the almost complete lack of symptoms at early stages and the absence of highly sensitive, effective and economical methods of screening for CRC, it is

\footnotetext{
*Corresponding author: Dr Francisco Javier Rodńguez-Berrocal, Departamento de Bioquímica, Genética e Inmunología, Universidad de Vigo, Facultad de Ciencias de Vigo; As Lagoas Marcosende, 36200 Vigo, Spain. Tel.: +34 986812571; Fax: +34 986812556; E-mail: berrocal@uvigo.es.
}

difficult to detect the carcinoma before the dissemination of the malignant tumour. Carcinoembryonic antigen (CEA) is the most commonly used serum tumour marker for CRC, but it is not recommended as screening or diagnostic test for this neoplasm, especially in the early stages [3-6]. At this time, the recommended screening methods for CRC are the faecal occult blood test (FOBT) and the sigmoidoscopy/colonoscopy with associated polypectomy [7,8]. Low rates of application of CRC screening methods have been attributed to resistance by physicians, patients, and the health care system [9]. Perhaps the problem is the high cost of applying those methods in screening campaigns for all the population with low or medium risk of CRC (i.e., healthy people over 50 years) [10]. Thus, it would be very useful to have one or several combined tumour markers that could help in the early detection of CRC. 


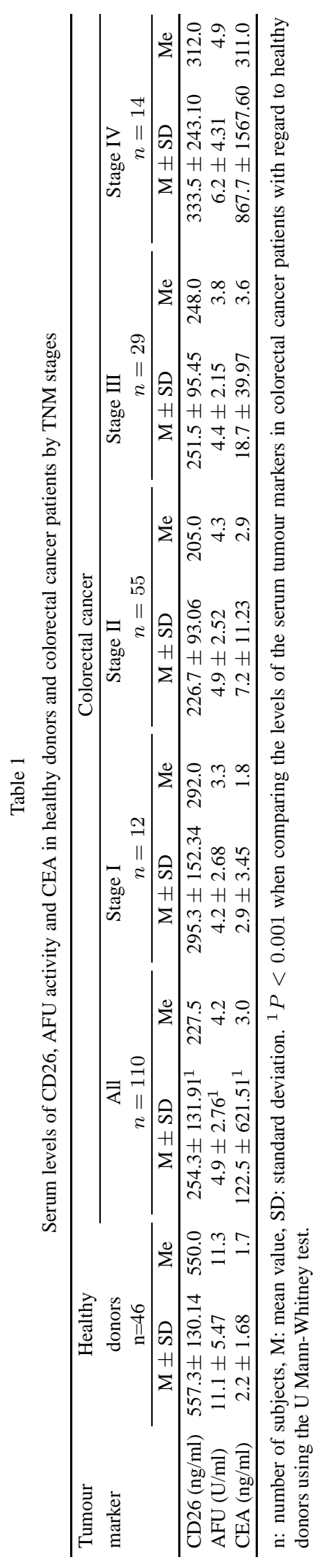


We have previously described the diagnostic value of two novel serum tumour markers: the soluble glycoprotein CD26 (dipeptidyl peptidase IV, EC 3.4.14.5.) and the alpha-L-fucosidase enzyme (EC 3.2.1.51) [11, 12]. A decrease in CD26 concentration and alpha-Lfucosidase activity (AFU) has been detected not only in serum of CRC patients but also in tissue of CRC tumours $[13,14]$ as well as in colorectal transformed cell lines $[15,16]$.

Here we show the results of measuring CD26 and AFU levels in sera of the same patients and donors in order to assess the diagnostic value of the combination of both markers. We have paid especial attention to the stages in which the tumour is not yet disseminated, in order to evaluate their usefulness as diagnostic or screening methods for CRC.

\section{Methods}

\subsection{Patients}

Preoperative blood and tumour samples were collected between January 1994 and December 1997 from 110 (54 females, 56 males) consecutive and potentially curable patients, operated on for CRC (74 colon, 36 rectum) at the Xeral-Cíes Hospital, Spain. All primary colorectal adenocarcinoma specimens were processed for regular pathological and histological examination. The grading of tumours was established according TNM classification [17]: 12 samples were classified as stage I, 55 as stage II, 29 as stage III and 14 as stage IV. Blood samples for the control group, consisting of 46 healthy blood donors ( 26 females, 20 males), were provided by the Galician Transfusion Centre. These controls were healthy and controlled donors. Most of them were habitual and some were new donors obtained in a campaign of the Galician Transfusion Centre at the University of Vigo. Blood from all these donors had been tested by the Galician Transfusion Centre.

\subsection{Preparation of samples}

The drawn blood was allowed to coagulate at room temperature and centrifuged at $2000 \times \mathrm{g}$ for $15 \mathrm{~min}-$ utes. The sera were stored at $-85^{\circ} \mathrm{C}$ until used, unless otherwise stated.

\subsection{CD26 assay}

The concentration of serum CD26 was analysed using specific immunoassays (human soluble CD26 ELISA Kit from Bender Medsystems, Vienna, Austria). ELISAs were performed according to the manufacturer's instructions: mean values of duplicated measurements were calculated and a sigmoid-shaped standard curve was determined by simultaneously analysing a dilution series of standard samples. A CD26 value lower than $410 \mathrm{ng} / \mathrm{ml}$ was considered as abnormal [11].

\subsection{Alpha-L-fucosidase assay}

AFU assays were always performed under linear conditions with respect to the amount of protein and the time of incubation. AFU activity was measured as previously described [12], using 4-methyl-umbelliferyl- $\alpha$ L-fucopyranoside (4-MU-fucoside) at a final concentration of $0.35 \mathrm{mM}$ as substrate and $100 \mathrm{mM}$ citric $\mathrm{acid} / \mathrm{K}_{2} \mathrm{HPO}_{4}$ buffer $\mathrm{pH}$ 5.0. Fluorescence was read on a Kontron fluorometer (using wavelengths of $365 \mathrm{~nm}$ and $460 \mathrm{~nm}$ for excitation and emission, respectively), and fluorescence values were corrected by subtracting the serum sample and the substrate blanks. One unit of enzyme (U) is defined as the amount of enzyme necessary to hydrolyse $1 \mathrm{nmol}$ of substrate per minute at $37^{\circ} \mathrm{C}$. An AFU activity value lower than $5.6 \mathrm{U} / \mathrm{ml}$ was considered as abnormal [12].

\subsection{Carcinoembryonic antigen assay}

The concentration of CEA was analysed by a commercial immunoassay method using the Enzymun-Test CEA (Boehringer Mannhein). This immunoassay is carried out in one step by means of a sandwich method using the streptavidine technology. A CEA value higher than $5 \mathrm{ng} / \mathrm{ml}$ was considered as abnormal [3].

\subsection{Calculation of the sensitivity, specificity and efficiency parameters}

Normal distribution was assessed using the Kolmogorov-Sminrov test. Variance homogeneity was evaluated by the Levene test. The statistical significance of the results was assessed using the U Mann-Whitney test performed in the SPSS software for Windows (Release 9.0).

The sensitivity was considered as the number of truepositives, and the specificity as the number of truenegatives, expressed as a percentage. The efficiency is 
the fraction of patients correctly classified, expressed in percentage.

When the tumour markers were used in combination, the sensitivity value was considered as the number of true-positives, and the specificity value as the number of true-negatives, detected by both tumour markers. Specific software made to calculate directly the sensitivity and the specificity of the tumour markers alone or combined was used.

\section{Results}

\subsection{CD26, alpha-L-fucosidase activity and carcinoembryonic antigen levels in serum from healthy donors and colorectal cancer patients}

Soluble CD26 concentration and AFU activity was determined in 46 sera from healthy donors and 110 sera from CRC patients. CEA concentration was also measured as an internal control of the study and to assess its diagnostic value in combination with CD26 and AFU. Means \pm standard deviations and medians of the tumour makers for donors and patients by TNM stages are shown in Table 1. CD26 and AFU activity levels were significantly lower $(P<0.001)$ in CRC patients than in healthy donors, whereas CEA levels were significantly higher $(P<0.001)$ in patients than in donors. Statistical differences of CD26, AFU and CEA levels between patients with colon versus patients with rectal tumours were not found.

\subsection{Diagnostic parameters of the combined use of CD26, alpha-L-fucosidase activity and carcinoembryonic antigen in the detection of colorectal cancer}

The diagnostic parameters of the combined use of CD26, AFU and CEA were established using a combination of two of the tumour markers in the detection of CRC. Table 2 shows the sensitivity, specificity and efficiency of the combination of CD26 and AFU, CD26 and CEA, and AFU and CEA in the diagnosis of CRC. The used values of cut-off were $410 \mathrm{ng} / \mathrm{ml}$ for CD26, $5.6 \mathrm{U} / \mathrm{ml}$ for $\mathrm{AFU}$ and $5.0 \mathrm{ng} / \mathrm{ml}$ for CEA, determined in previous studies $[3,11,12]$.

The combination of CD26 and AFU in the diagnosis of CRC showed a sensitivity of $64 \%$, with a specificity of $100 \%$ and an efficiency of $82 \%$. When CD26 and CEA were used in combination, the diagnostic sensitivity was lower $(34 \%)$ with a specificity of $100 \%$ and an efficiency of $67 \%$. The combination of AFU and CEA showed the lowest sensitivity $(25 \%)$ with a specificity of $100 \%$ and an efficiency of $63 \%$.
Table 2

Diagnostic parameters of the combined use of CD26, AFU activity and CEA in the detection of colorectal cancer

\begin{tabular}{lccc}
\hline Tumor marker & Sensitivity & Specificity & Efficiency \\
\hline CD26 and AFU & 64 & 100 & 82 \\
CD26 and CEA & 34 & 100 & 67 \\
AFU and CEA & 25 & 100 & 63 \\
\hline
\end{tabular}

CD26 cut-off: $410 \mathrm{ng} / \mathrm{ml}$; AFU cut-off: $5.6 \mathrm{U} / \mathrm{ml}$; CEA cut-off: $5.0 \mathrm{ng} / \mathrm{ml}$. Sensitivity, specificity and efficiency values were calculated as explained in methods, and are expressed in percentage.

Table 3

Diagnostic sensitivity at the $100 \%$ of specificity of the combined use of CD26, AFU activity and CEA in the detection of colorectal cancer patients classified by TNM stages

\begin{tabular}{lcccc}
\hline & \multicolumn{4}{c}{ TNM stage } \\
\cline { 2 - 5 } & I & II & III & IV \\
\hline $\begin{array}{l}C D 26 \text { and } A F U \\
\text { Sensitivity }\end{array}$ & 75 & 66 & 66 & 43 \\
$\begin{array}{l}C D 26 \text { and } C E A \\
\text { Sensitivity }\end{array}$ & 17 & 27 & 35 & 71 \\
$\begin{array}{l}\text { AFU and CEA } \\
\text { Sensitivity }\end{array}$ & 17 & 15 & 31 & 57 \\
\hline
\end{tabular}

CD26 cut-off: $410 \mathrm{ng} / \mathrm{ml}$; AFU cut-off: $5.6 \mathrm{U} / \mathrm{ml}$; CEA cut-off: $5.0 \mathrm{ng} / \mathrm{ml}$. Sensitivity values were calculated as explained in methods and are expressed in percentage.

\subsection{Diagnostic sensitivity of the combined use of CD26, alpha-L-fucosidase activity and carcinoembryonic antigen in the detection of colorectal cancer patients classified by TNM stages}

The diagnostic sensitivities of the combinations of the three tumour markers were also evaluated by each TNM stage of the tumours (Table 3).

The results showed that the combined use of CD26 and AFU gave a sensitivity of $75 \%$ in stage I, $66 \%$ in stages II and III and $43 \%$ in stage IV. The combination of CD26 and CEA showed poor sensitivity levels in the stages I (17\%), II (27\%) and III (35\%) and higher sensitivity in the stage IV $(71 \%)$. The combined use of AFU and CEA also showed poor diagnostic sensitivity levels in the stages I (17\%), II (15\%) and III (31\%), whereas the diagnostic sensitivity in the stage IV was $57 \%$.

Thus, the combination of CD26 and AFU showed the best sensitivity and specificity in the diagnosis of patients at non-disseminated stages.

\section{Discussion}

We have previously reported significant decreases of more than $50 \%$ in the CD26 concentration and AFU 
activity of serum from CRC patients, establishing the sensitivity and specificity of each novel marker used alone in the diagnosis of CRC [11,12]. The decrease of serum CD26 and AFU could be related with the diminution of the levels of these proteins in CRC cells [1316]. However, the impairment of more than $50 \%$ in serum CD26 and AFU activity in CRC does not seem to be only originated by alterations of these proteins in tumour cells. The decreased levels of CD26 could be related with an immune defective antitumour response in CRC. For instance, a defect in interleukin-12 production [18] that is a well-known CD26 up-regulator [19] has been described in CRC. The decreased levels of AFU activity could be related with the maintenance of the observed higher levels of fucosylation in tumour cells [20].

In the present paper we have analysed if the diagnostic value of CD26 and AFU is improved when both markers are used together in the detection of CRC. On the other hand, we have also analysed if the results of CEA in the detection of CRC are improved when it is used in combination with CD26 or AFU. Normal levels of each tumour marker were established using as reference the previously described cut-off values: $410 \mathrm{ng} / \mathrm{ml}$ for CD26 [11], $5.6 \mathrm{U} / \mathrm{ml}$ for AFU [12] and $5.0 \mathrm{ng} / \mathrm{ml}$ for CEA [3]. Sensitivity, specificity and efficiency values obtained in the present study for CD26, AFU and CEA, when these markers were used alone in the diagnosis of CRC, were very similar to those previously reported [11,12].

When CD26 is used alone at the $100 \%$ of specificity, sensitivity was of $33 \%$ in the stage I, $65 \%$ in the stage II and $66 \%$ in the stage III [11]. These values for AFU were even lower [12]. In this work we have demonstrated that the combined use of CD26 and AFU showed good sensitivity levels $(64 \%)$ at the highest specificity $(100 \%)$. Furthermore, the sensitivity at nondisseminated stages was of $75 \%$ in stage I and $66 \%$ in stages II and III, higher than that observed in the stage IV (43\%). However, the combination of CD26 or AFU with CEA offers low sensitivity levels, especially in the non-disseminated stages. These results confirm that CEA is not useful in the diagnosis of CRC.

FOBT screening procedure has been demonstrated to reduce the risk of death of CRC [21-24] and it is the most commonly performed screening test for CRC. It includes an analysis of three serially obtained samples [21-23] and when any of the three tests for each sample is positive, colonoscopy is recommended [25]. We propose similar studies with CD26, AFU, or both, combined with FOBT to assess if the detection rates of
CRC could be improved. Other studies have recently been proposed in order to improve the results obtained with FOBT, through the detection of DNA alterations 'such as APC or K-ras mutation' in stool samples [26, 27]. The advantages of the methods that we here propose are that they are easy and inexpensive.

In conclusion, our findings provide a new tool especially sensitive in the diagnosis of CRC patients in stage I, but also in stages II and III. The results of the combination of these markers appear promising, and merit further study in a screening setting in order to assess if they could be used alone or combined with other screening tests -such as FOBT- to help clinicians to decide if healthy people with low or medium risk of CRC should undergo other more invasive methods, such as sigmoidoscopy/colonoscopy, which confirm or discard the presence of the tumour.

\section{Acknowledgements}

We thank Ms. Beatriz Otero for her excellent technical assistance. We also thank "Centro de Transfusión de Galicia" and "Hospital Xeral-Cíes de Vigo" for providing samples. This work was supported by grants from "Xunta de Galicia” (PGIDT00PXI30115PR) and "Universidad de Vigo". Daniel Ayude was supported by a postdoctoral contract of the "Programa Torres Quevedo, M.C.Y.T.”.

\section{References}

[1] http://www.iarc.fr, International Agency for Research on Cancer. Cancer incidence and mortality data bases, last accessed 1 July, 2003.

[2] S. Srivastava, The Early Detection Research Network Second Annual Scientific Workshop 14-16 October 2001, Seatle, Washington, USA, Dis. Markers 18 (2002), 1.

[3] American Society of Clinical Oncology, Clinical practice guidelines for the use of tumour markers in breast and colorectal cancer, J. Clin. Oncol. 14 (1996), 2843-2877.

[4] C.E. Desch, A.B. 3rd Benson, T.J. Smith, P.J. Flynn, C. Krause, C.L. Loprinzi, B.D. Minsky, N.J. Petrelli, D.G. Pfister and M.R. Somerfield, Recommended colorectal cancer surveillance guidelines by the American Society of Clinical Oncology, J. Clin. Oncol. 17 (1999), 1312-1321.

[5] A.B. 3rd Benson, C.E. Desch, P.J. Flynn, C. Krause, C.L. Loprinzi, B.D. Minsky, N.J. Petrelli, D.G. Pfister, T.J. Smith and M.R. Somerfield, 2000 update of American Society of Clinical Oncology colorectal cancer surveillance guidelines, J. Clin. Oncol. 18 (2000), 3586-3588.

[6] R.C. Jr Bast, P. Ravdin, D.F. Hayes, S. Bates, H. Jr Fritsche, J.M. Jessup, N. Kemeny, G.Y. Locker, R.G. Mennel and M.R. Somerfield, 2000 update recommendations for the use of tumor markers in breast and colorectal cancer: clinical practice guidelines of the American Society of Clinical Oncology, $J$. Clin. Oncol. 19 (2001), 1865-1878. 
[7] J.W.W. Coebergh, Challenges and pitfalls of mass-screening in the European Union, Eur. J. Cancer 36 (2000), 1469-1472.

[8] Advisory Committee on Cancer Prevention, Recommendations on cancer screening in the European Union, Eur. J. Cancer 36 (2000), 1473-1478.

[9] S.W. Vernon, Participation in colorectal cancer screening: a review, J. Natl. Cancer Inst. 89 (1997), 1406-1422.

[10] D.F. Rasnsohoff and R.S. Sandler, Screening for colorectal cancer, N. Engl. J. Med. 346 (2002), 40-44.

[11] O.J. Cordero, D. Ayude, M. Nogueira, F.J. Rodríguez-Berrocal and M. Páez de la Cadena, Preoperative serum CD26 levels: diagnostic efficiency and predictive value for colorectal cancer, Br. J. Cancer 83 (2000), 1139-1146.

[12] D. Ayude, J. Fernández-Rodríguez, F.J. Rodríguez-Berrocal, V.S. Martínez-Zorzano, A. de Carlos, E. Gil and M. Páez de la Cadena, Value of the serum $\alpha$-L-fucosidase activity in the diagnosis of colorectal cancer, Oncology 59 (2000), 310-316.

[13] J. Ten Kate, J.T. Wijnen, J. Boldwijn, P.M. Khan and F.T. Bosman, Immunohistochemical localization of adenosine deaminase complexingn protein in intestinal mucosa and in colorectal adenocarcinoma as a marker for tumour cell heterogeneity, Histochem. J. 17 (1985), 23-31.

[14] M. Páez de la Cadena, J. Fernández, A. de Carlos, V.S. Martínez-Zorzano, E. Gil-Martín and F.J. Rodríguez-Berrocal, Low levels of $\alpha$-L-fucosidase activity in colorectal cancer are due to decreased amounts of the enzymatic protein and are related with Dukes' stage, Int. J. Oncol. 9 (1996), 747-754.

[15] J. Ten Kate, H.F. van den Ingh, P.M. Khan and F.T Bosman, Adenosine deaminase complexing protein (ADCP) immunoreactivity in colorectal adenocarcinoma, Int. J. Cancer 37 (1986), 479-485.

[16] A. Merino-Trigo, F.J. Rodríguez-Berrocal, E. de Miguel and M. Páez de la Cadena, Activity and properties of alpha-Lfucosidase are dependent on the state of enterocytic differentiation of HT-29 colon cancer cells, Int. J. Biochem. Cell Biol. 34 (2002), 1291-1303.

[17] P. Hermanek and L.H. Sobin, UICC TNM Clasification of the malignant tumours: Colon and Rectum, 4th edition, 2nd revision, Springer, London, 1992, pp. 52-56.

[18] R.J. O'Hara, J. Greenman, P.J. Drew, A.W. McDonald, G.S. Duthie, P.W. Lee and J.R. Monson, Impaired interleukin-12 production is associated with a defective anti-tumour response in colorectal cancer, Dis. Colon Rectum 40 (1998), 460-463.

[19] O.J. Cordero, F.J. Salgado, J.E. Viñuela and M. Nogueira, Interleukin-12 enhances CD26 expression and dipeptidyl peptidase IV function on human activated lymphocytes, Immunobiology 197 (1997), 522-533.

[20] J. Fernández Rodríguez, M. Páez de la Cadena, V.S. MartínezZorzano and F.J. Rodríguez-Berrocal, Fucose levels in sera and in tumours of colorectal adenocarcinoma patients, Cancer Lett 121 (1997), 147-153.

[21] J.S. Mandel, J.H. Bond, T.R. Church, D.C. Snover, G.M. Bradley, L.M. Schuman and F. Ederer, Reducing mortality from colorectal cancer by screening for fecal occult blood. Minnesota Colon Cancer Control Study, N. Engl. J. Med. 328 (1993), 1365-1371.

[22] O. Kronborg, C. Fenger, J. Olsen, O.D. Jorgensen and O. Sondergaard, Randomised study of screening for colorectal cancer with faecal-occult-blood test, Lancet $\mathbf{3 4 8}$ (1996), 14671471.

[23] J.D. Hardcastle, J.O. Chamberlain, M.H. Robinson, S.M. Moss, S.S. Amar, T.W. Balfour, P.D. James and C.M. Mangham, Randomised controlled trial of faecal-occult-blood screening for colorectal cancer, Lancet 348 (1996), 14721477.

[24] Y. Niv, M. Lev-El, G. Fraser, G. Abuksis and A. Tamir, Protective effect of faecal occult blood test screening for colorectal cancer worse prognosis for screening refusers, Gut 50 (2002), 33-37.

[25] D.F. Ransohoff and C.A. Lang, Screening for colorectal cancer with the fecal occult blood test: a background paper, Ann Intern Med 126 (1997), 811-822.

[26] G. Traverso, A. Shuber, B. Levin, C. Johnson, L. Olsson, D.J. Jr Schoetz, S.R. Hamilton, K. Boynton, K.W. Kinzler and B. Vogelstein, Detection of APC mutations in fecal DNA from patients with coloretal tumors, N. Engl. J. Med. 346 (2002), 311-320.

[27] Y. Ito, S. Kobayashi, T. Taniguchi, O. Kainuma, T. Hara and T. Ochiai, Frequent detection of K-ras mutation in stool samples of colorectal carcinoma patients after improved DNA extraction: Comparison with tissue samples, Int. J. Oncol. 20 (2002), 1263-1268. 


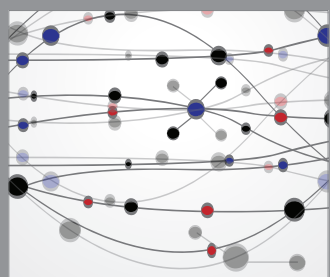

The Scientific World Journal
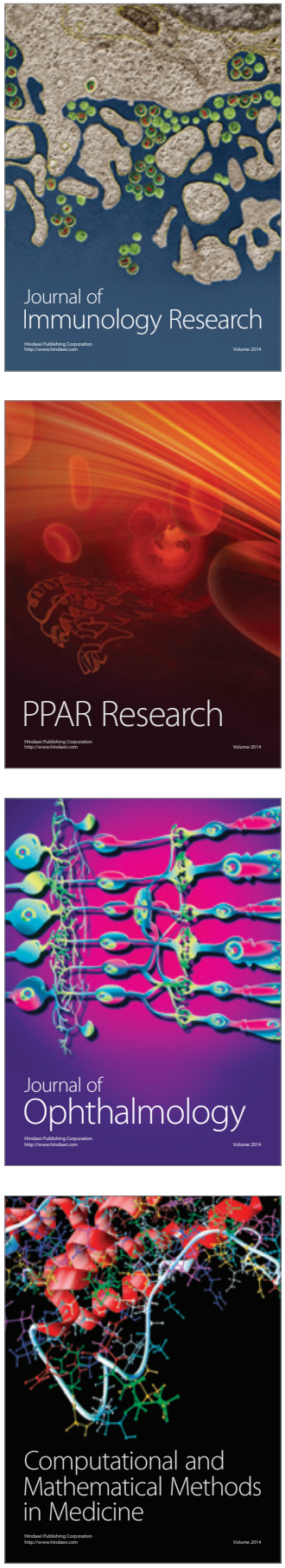

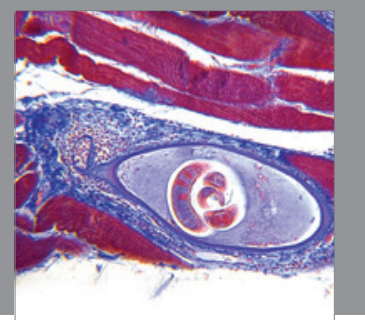

Gastroenterology

Research and Practice
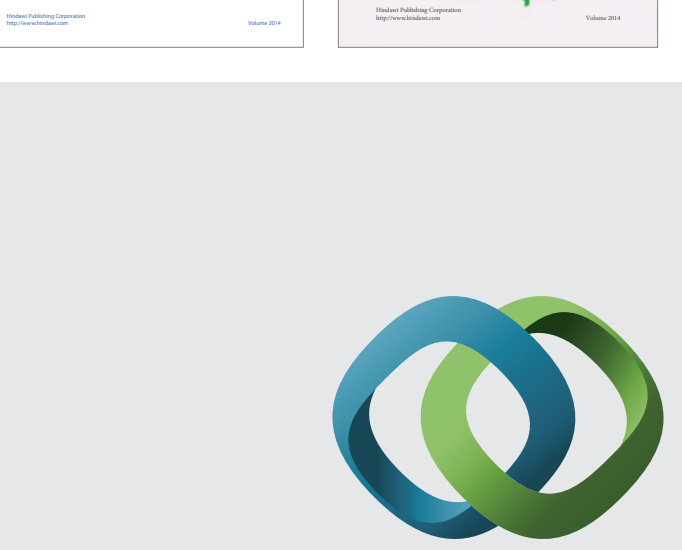

\section{Hindawi}

Submit your manuscripts at

http://www.hindawi.com
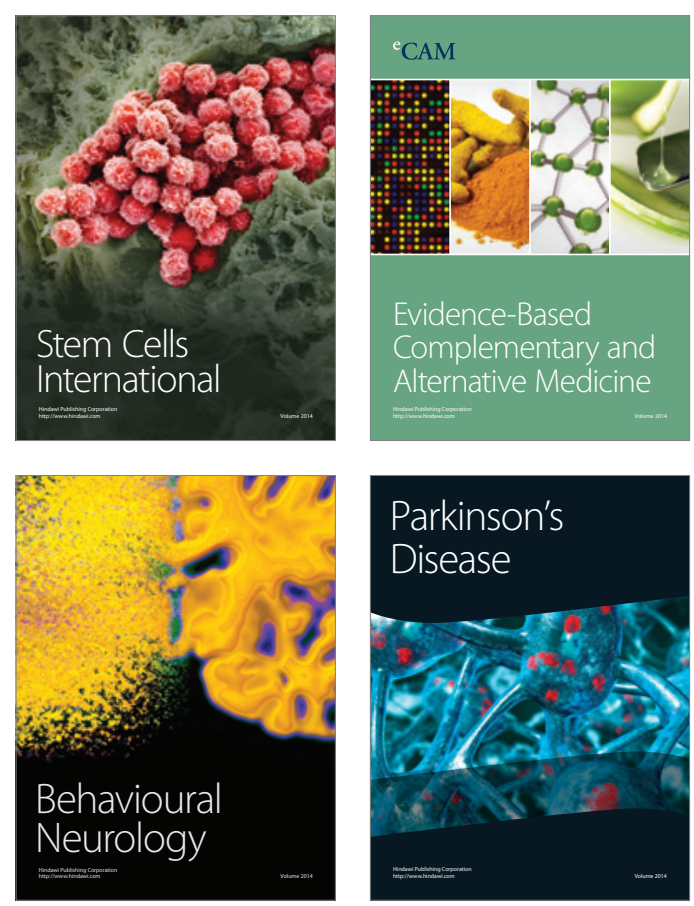

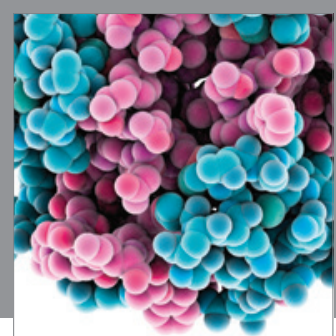

Journal of
Diabetes Research

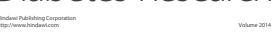

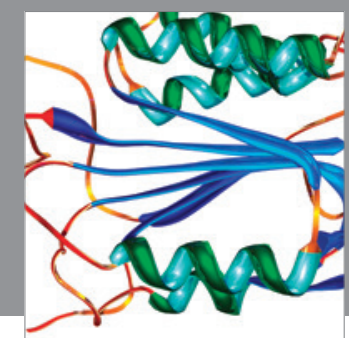

Disease Markers
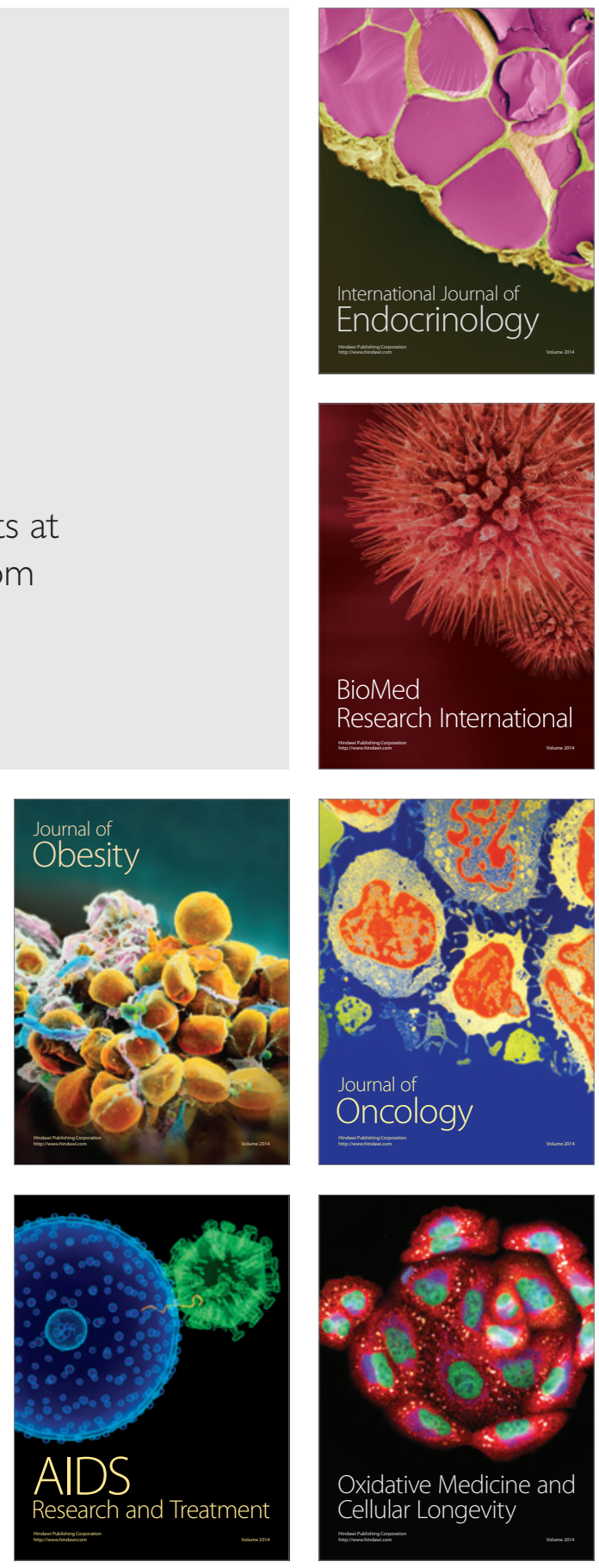